\title{
Guemil: Diseño y medición de significados de un set de íconos para representar el riesgo y la emergencia
}

\section{Guemil: Design and measuring on meanings of a set of icons to represent risk and emergency}

\author{
Rodrigo Ramírez \\ Pontificia Universidad Católica, Chile \\ rramireo@uc.cl
}

\begin{abstract}
Oriented to discuss about how information contributes to resilience, this paper presents a research-based project that integrates design and performance measuring. This project is Guemil, an open source initiative aimed to represent risk and emergency contexts through icons. As a simple solution with a focus on risk management cycle + user experience, the set of icons is designed around a 'before > during > after' concept. To verify performance about interpretation from users, icons are currently being tested worldwide. Reflections from the process and future projections are presented as conclusion.
\end{abstract}

Keywords: Información; emergencia; íconos; herramientas gráficas; desempeño.

\section{Introducción}

La información, entendida como un mensaje efectivo es parte central de la comunicación visual y con múltiples definiciones dependiendo de su enfoque de origen: La disciplina del Diseño de Información es definida por Horn (2000) como el 'arte y ciencia de presentar información para que pueda ser usada por humanos con eficiencia y efectividad'. Por sus alcances, se trata también de un ámbito multi-dimensional en su aplicación (Pettersson, 2002) que en la llamada 'era de la información', forma parte central de lo cotidiano para personas y organizaciones.

En esta multiplicidad y más allá de solo visualizarla, el procesamiento óptimo de información puede también afectar positivamente la experiencia de las personas en contextos físicos o virtuales, permitiendo interpretar y actuar, optimizando la transferencia de conocimiento y facilitando las decisiones (Allard, Briones, et al., 2014).
Como recurso del lenguaje, la información visual puede ser sistemática en su despliegue, contando hoy con diversas aplicaciones gracias a las tecnologías de información. Uno de estos recursos, los sistemas de íconos o pictográficos forman parte de la vida cotidiana de las personas. El lenguaje de íconos se utiliza para representar información normalizada, multiplataforma y entendible de modo rápido, asociado a menudo a las necesidades de usuarios en espacios públicos o en la interacción con tecnologías, constituyendo una lingua franca de nuestro tiempo (Zender \& Mejía, 2013). Como lo ilustra la Figura 1, una de las aspiraciones de este lenguaje normalizado, ha sido la de llegar a conformar en el ámbito global, un código multi-cultural e incluso universal.
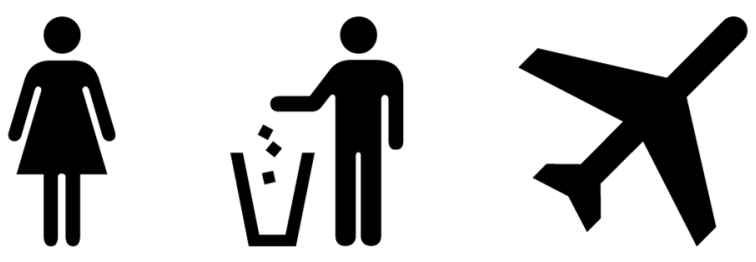
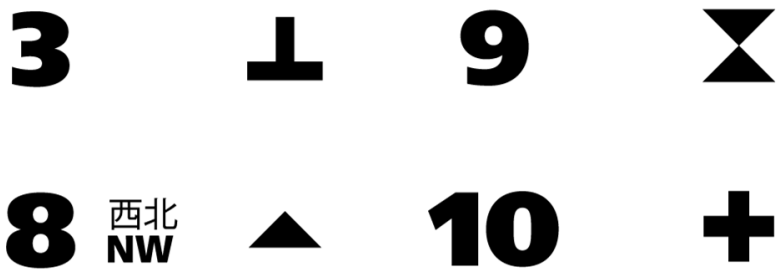

Figura 1: Símbolos públicos, estándar AIGA DOT (1979) + Señales sobre intensidad de tifones, Hong Kong Observatory (1973) 
Sin embargo, el ideal de 'universalidad' de la información representada en iconos depende de dos variables prácticas: (1) El contexto en el que se presenta la información y (2) cómo completa el mensaje una persona, qué interpreta.

También y como indica Jaenichen (2011), lo que podría resultar natural de comprender en lo cotidiano se transforma radicalmente en situaciones disruptivas o extremas. En éstas las condiciones para el procesamiento de información son otras: El contexto normal es alterado y para las personas la necesidad de información -lo que requiere saber en cada momento- puede variar dramáticamente.

Observar lo que ocurre en contextos cotidianos es natural para una disciplina centrada en las personas como el diseño. Entender además las carencias que se generan ante un escenario disruptivo, poniendo el foco en qué se puede evitar, en cómo prepararse ante un evento potencial o mitigar ante una amenaza, puede contribuir a manejar y reducir la incertidumbre en las comunidades. En esto la información es un aporte fundamental contribuyendo a construir una experiencia resiliente.
Por sus características geográficas y geológicas como país, Chile presenta condiciones excepcionales en el mundo, las que enfrentan permanentemente a las personas a situaciones de riesgo, amenaza o desastre. En este contexto, en general la necesidad de informar suele abordarse más desde la indicación y el soporte (señalización por ejemplo) aunque menos desde entender qué tipo de indicaciones es necesario desplegar para cada momento, lo que podría estar más en sintonía con la experiencia de las personas y lo que requieren. Teóricamente, el modelo conocido como Ciclo de Gestión del Riesgo de Desastres (DRMC), como el desarrollado por TorqAid (2016) y que muestra la figura 2, permite observar este proceso de una forma distinta -basada en etapas o momentos de la experiencia-.

En 2010 se detecta la oportunidad de abordar la emergencia como un problema de diseño. Esta se comienza a trabajar académicamente desde la Escuela de Diseño UC, explorando soluciones de comunicación visual para tal contexto. Aquí, otra de las oportunidades generadas es la de desarrollar investigación basada en proyectos, integrando disciplinas, organizaciones e investigadores que trabajan en la emergencia.

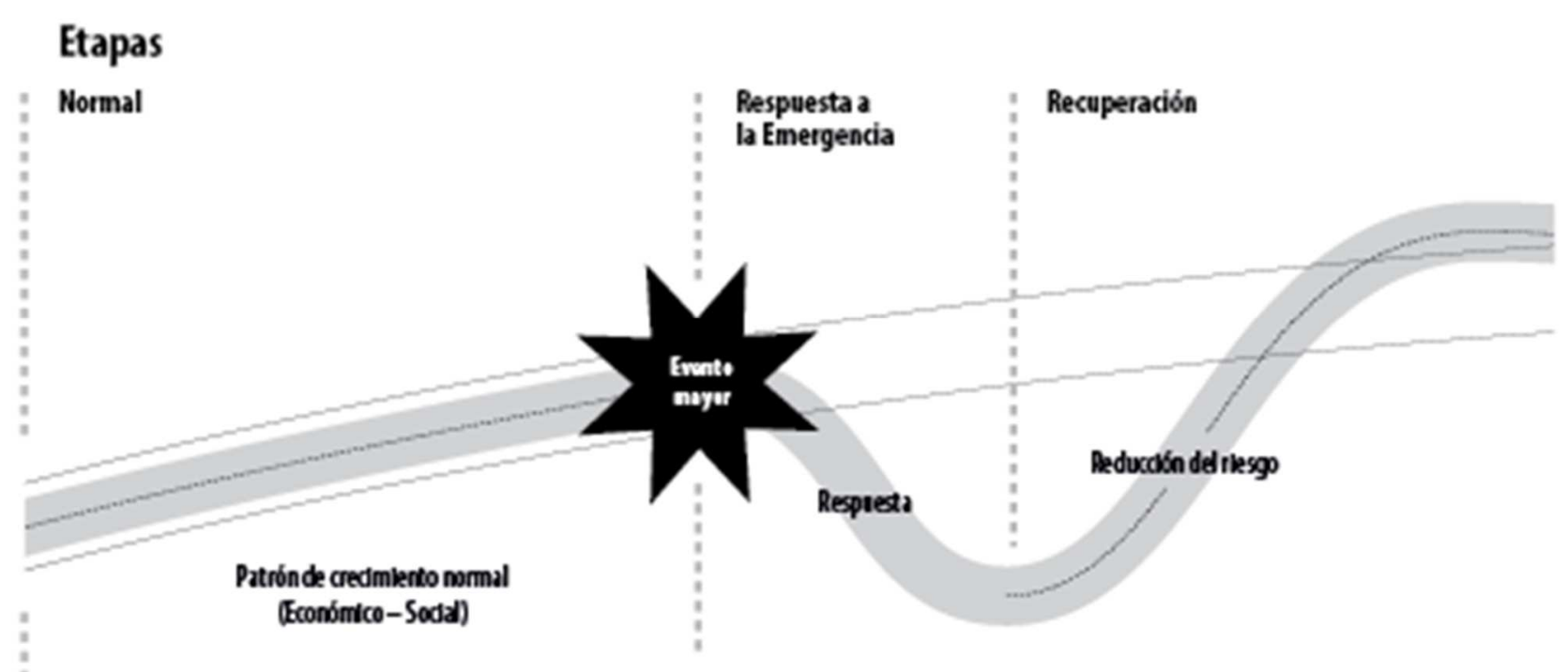

Antes

Durante

Después

The Disaster Risk Management Cyde, DRMC

Figura 2: Adaptación del Disaster Risk Management Cycle, DRMC Framework (2016)

Chris Piper/TorqAid (C) 2002 - 2017 DRMC, licenciado bajo una Creative Commons International License 


\section{Un lenguaje normalizado para la emergencia}

El caso a presentar es el proyecto Ilamado Guemil; una iniciativa basada en íconos para representar situaciones de riesgo y emergencia. Los iconos son empaquetados como fuente tipográfica de acceso abierto y distribución gratuita a través de la web. La figura 3, muestra algunos de los 96 íconos desarrollados en la versión 0.5b, 2016.

En términos generales, su diseño fue pensado para desplegar elementos de información visibles y fácilmente entendibles. Este considera un lenguaje gráfico normalizado, analógico [más icónico que simbólico, ver fig 4], donde puedan verse representadas de forma simple, situaciones y/o acciones ante una emergencia. Otra decisión del diseño fue hacerla accesible en su aplicación, como fuente tipográfica digital open source, para computadores y web. También es importante mencionar dos atributos como innovación:
(1) Posibilita abordar la emergencia desde un esquema de acceso abierto, facilitando su adopción. Por ejemplo al complementar otros lenguajes como el texto, o adaptándose a la realidad socio-geográfica y también a las prácticas locales de manejo de emergencias;

(2) Es una solución visual para situaciones, acciones e información donde es posible adaptar las representaciones a diversos soportes, tecnologías y contextos de información, ampliando su alcance a otros idiomas y/o culturas.

Guemil es uno de los proyectos fundadores del Design Network for Emergency Management (dnem.org). Para dar cuenta del rol que puede tener el diseño para la emergencia, ha sido presentado en diversos eventos, y fue reconocido como 'Shortlist' en los IIIDaward 2017, del International Institute for Information Design. Esto demuestra el interés y eventual impacto que este tipo de iniciativas llegan a tener globalmente, constituyendo un potencial recurso efectivo para reconocer riesgos y planear la comunicación antes de la emergencia.
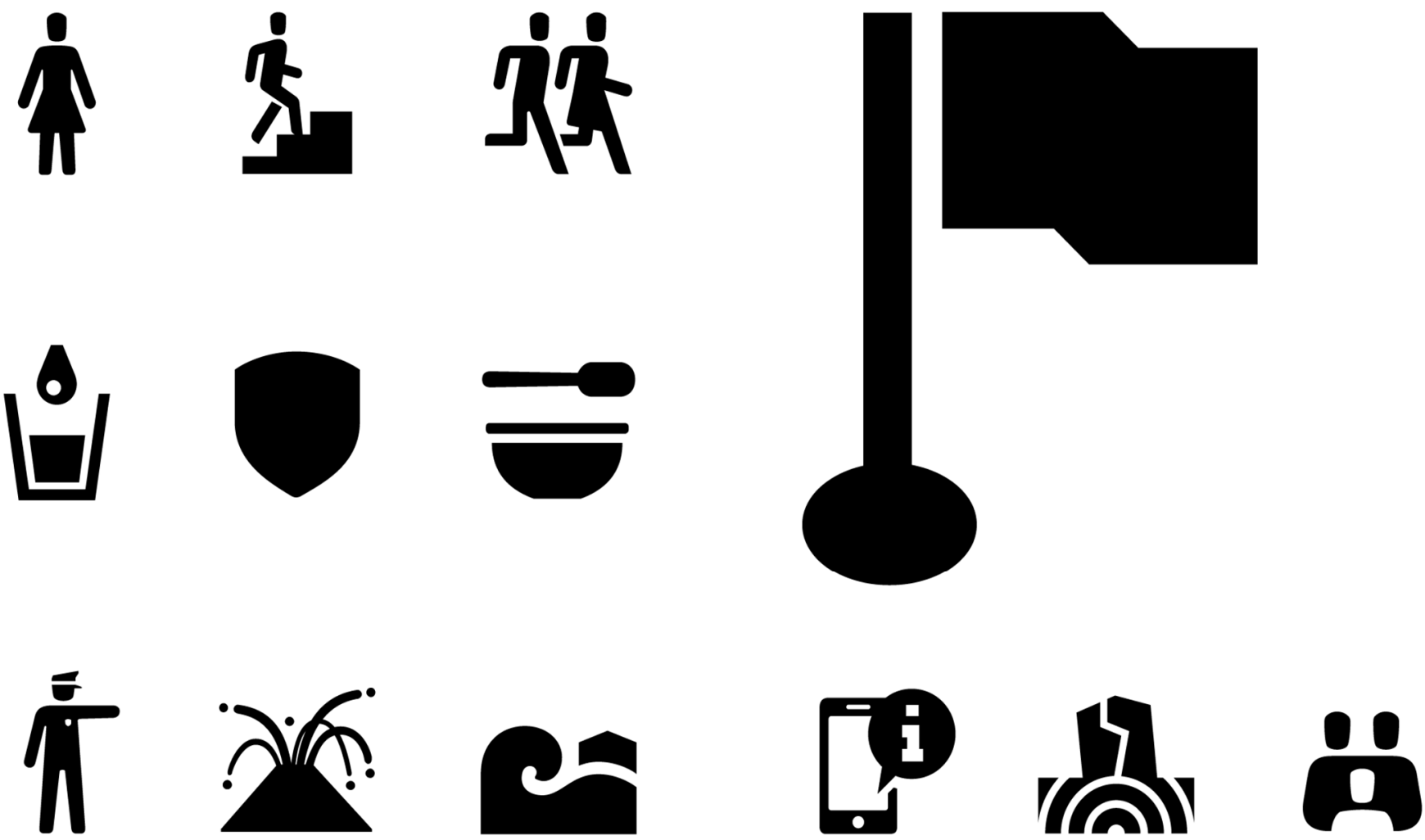

Figura 3: Algunos de los íconos Guemil, v 0.5b. El set de íconos es descargable desde el sitio www.guemil.info. 


\section{¿Íconos, pictogramas, símbolos?}

Como elementos gráficos sintetizados, Guemil dispone un set para representar diversas etapas: antes $\rightarrow$ durante $\rightarrow$ después de una emergencia, abarcando el ciclo de riesgo completo. Sin embargo, ¿cuáles de estos son íconos, símbolos, pictogramas? Los tres términos se utilizan indistintamente para referirse a estos 'elementos portadores de significado', como los definen Abdullah y Hüber (2006). La figura 4 grafica las diferencias comúnmente acordadas, y cómo el set incorpora en su diseño diversas tipologías. Estos pueden representar personas, acciones, riesgos, situaciones, dependiendo de su rol como elemento de información.

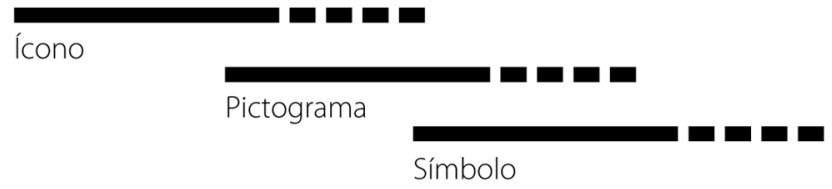

Representación analógica

Representación convenida

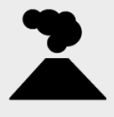

ĺcono

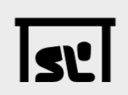

Pictograma

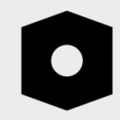

Símbolo
Figura 4: Diferencias fundamentales entre los tres términos: ícono + pictograma + símbolo y cómo son aplicados en Guemil.

Diseñado y disponible el set de íconos, uno de los desafíos es medirlo como herramienta gráfica con asignaciones precisas de significado y validar su desempeño. Ello se desarrolla a continuación.

\section{Medir los íconos: Significado + Diferencias}

Como se indica, Guemil es un recurso gráfico simple para desplegar información en etapas y contribuir a la experiencia de las personas en escenarios críticos. El diseño de los íconos considera también un acceso + aplicación abierta. Hasta acá su adopción inicial ha sido principalmente en plataformas de información (ej. interfaces de usuario) aunque también otras pueden ser consideradas (ej. material para la preparación). La colaboración y divulgación de distintas organizaciones ha resultado fundamental (CIGIDEN, 2016; DNEM, 2016).

Más allá de lo visual sin embargo, por la naturaleza funcional de la solución se hizo también necesario conocer cómo contribuye efectivamente a la comunicación, buscando identificar qué se entiende -y qué no- en las representaciones. Se desarrollaron algunos principios metodológicos junto al prototipado de tests, observando prácticas internacionales relevantes (ISO 2011; ANSI 2011) y se estableció medir fundamentalmente dos variables en el diseño visual de los íconos: Significado y Comprensión.

De esta forma, se seleccionaron 72 de los 96 íconos para testear. Se diseñaron pruebas para establecer su desempeño sobre el significado y diferencias a los que se agregaron algunas preguntas sobre desempeño. El conjunto se sumó a la plataforma web como formulario interactivo bilingüe -inglés y español-. La plataforma actualmente disponible -en proceso de alcanzar las 200 respuestas por ícono-, y que se muestra en la figura 5, contabiliza hasta Julio 2017 más de mil respuestas totales desde diversos lugares del mundo, tras ocho meses online.
Significados

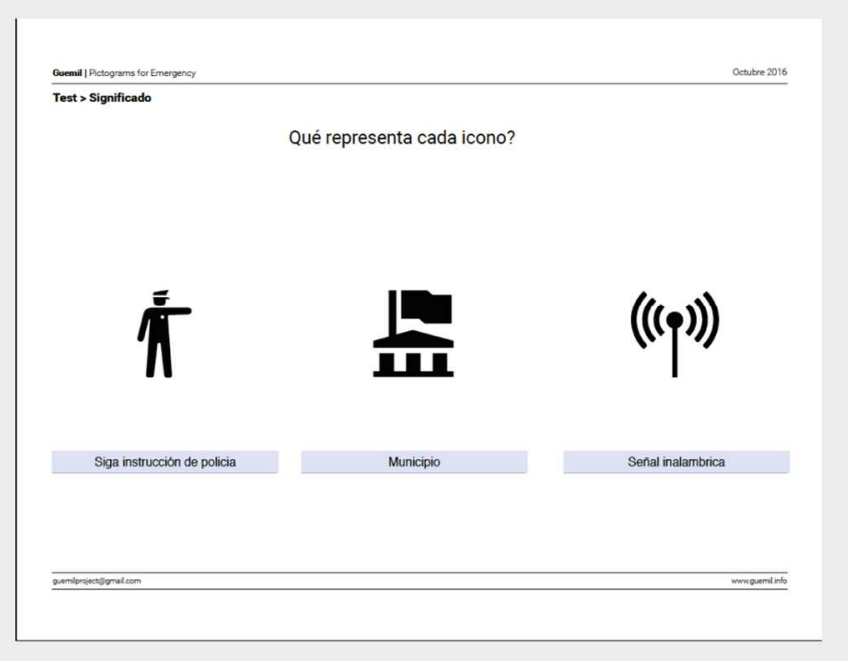

Diferencias

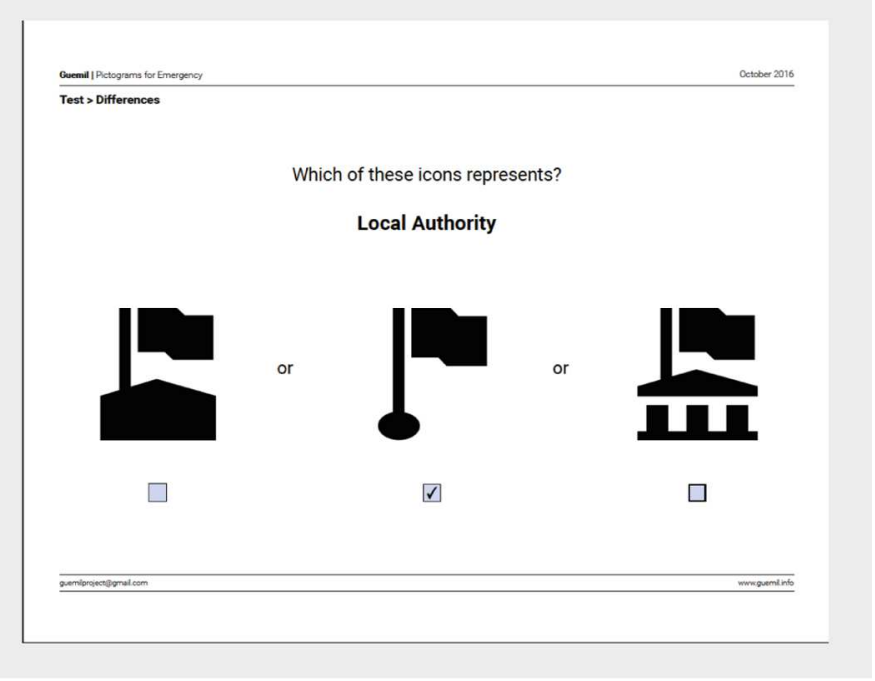

Figura 5: Muestras de los test Guemil (formularios interactivos), con preguntas sobre significados y diferencias (estos pueden descargarse desde el sitio www.test.guemil.info). 


\section{Cómo se mide el desempeño en significados}

Una vez desarrollada la versión inicial de los íconos y para levantar indicadores de desempeño se desarrolló un kit de testeo, el que se ha implementado hasta acá en formularios interactivos de respuesta abierta. Las pruebas han sido recogidas y procesadas anónimamente aplicando una tabla de ponderación de las respuestas en base a seis valores, basándose en el desarrollo que tanto Brugger (1999), Olygay (2001) o Frascara (2011) sugieren como procedimiento para evaluar símbolos para uso público (ISO, 2007) y para ámbitos asociados a la seguridad en el trabajo (ISO, 2011, ANSI, 2011). Los valores de la escala fueron adaptados y se muestran en la tabla 1.
Tabla 1: Escala denominada 'Brugger-Frascara' adaptada a seis valores, que se aplica al ponderar las respuestas de los tests

\begin{tabular}{|c|c|c|c|c|c|c|}
\hline Puntaje & 1 & 2 & 3 & 4 & 5 & 6 \\
\hline Significado & Correcto & $\begin{array}{c}\text { Semi- } \\
\text { correcto }\end{array}$ & Ambiguo & Incorrecto & Opuesto & $\begin{array}{l}\text { Sin } \\
\text { respuesta }\end{array}$ \\
\hline
\end{tabular}

El proceso se completa con una ponderación de los resultados, la que arroja un porcentaje asociado al desempeño de cada ícono. Las pruebas iniciales, publicadas en la web desde Mayo 2017, permiten visualizar resultados sobre este desempeño, algunos de los cuales se ilustran en la figura 6 . Ver también las notas metodológicas a continuación.

\section{Base de datos con respuestas $\quad$ Visualización de resultados}

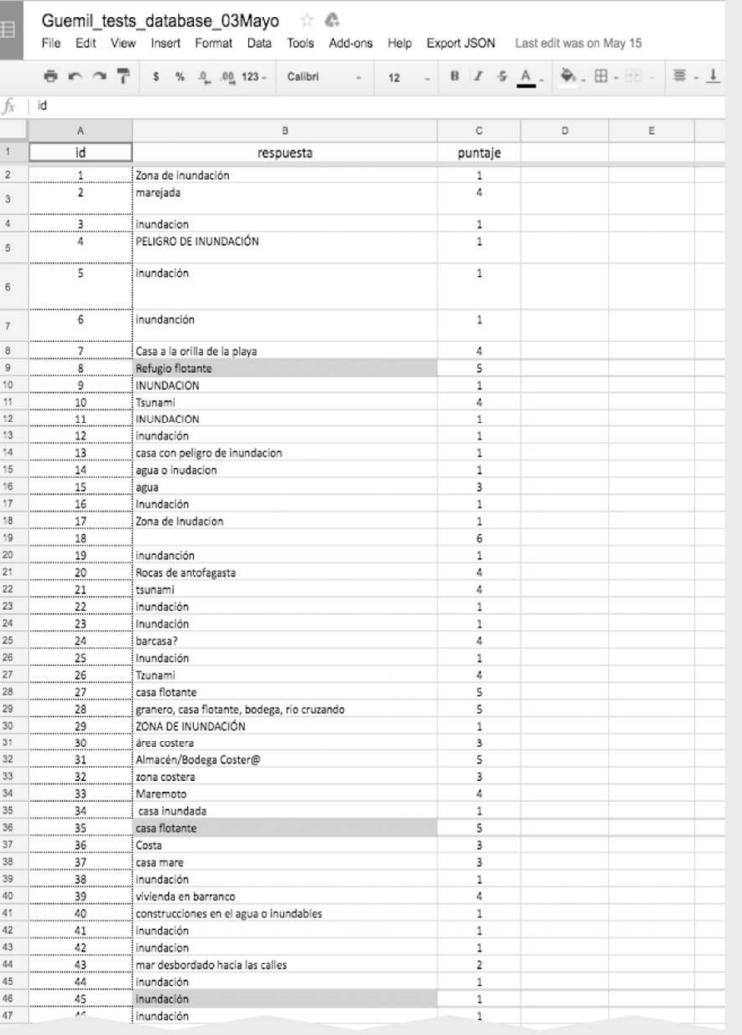

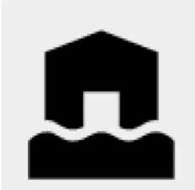

$72 \%$

Performance

Desempeño

51_Flood

Answers Respuestas

120

correct

Correcta

\section{5}

Almost

correct

Casicorrecta

20

Incorrect

Incorrects

9

Opposite

Opuesto
187

12

No answe

$\mathrm{Sin}$

respuesta

4 Índice

de desempeño

21

Doubtiul

Dudosa

12
No answer
Sin
respuesta

Detalle de las respuestas

www.test.guemil.info

Figura 6: Ejemplo de la visualización de resultados del testeo del ícono 51_Flood, con su ponderación de resultados a Mayo 2017 (187 respuestas). Todos los resultados pueden verse en la web del proyecto. 


\section{Notas metodológicas sobre el desempeño de significados, con comentarios del proceso}

La visualización de los resultados permite formarse algunas ideas iniciales sobre qué dicen sobre la representación simbólica y cómo las personas interpretan el ámbito de la emergencia. Ambos son aspectos interesantes como generación de conocimiento en diseño y referencia además para la investigación basada en proyectos.

- Se ha dispuesto cada icono sin sugerir significados, para testear su comprensión desde la pregunta: ¿Qué representa cada ícono? Siendo respuestas abiertas, cada una ha sido ponderada según la tabla de evaluación de seis valores (presentada en este artículo como tabla 1).

- La práctica internacional (ISO, 2011) establece disponer de 200 respuestas válidas por ícono. Otros estándares (ANSI Z353.3, 2011) establecen un rango mínimo de 50 respuestas para validar. En el caso de Guemil hasta Mayo 2017, la mayor cantidad de respuestas por ícono es de 187 y la mínima 99.

- De acuerdo a estas mismas prácticas de desempeño (Frascara 2011, ISO 7010:2011), un ícono aceptado para contextos de emergencia requiere un mínimo de $83 \%$ de respuestas apropiadas para validar su uso, ver figura 7. (Para el caso de símbolos públicos, este índice es de 66\%).

- Se considera que los íconos bajo el $83 \%$ de desempeño debieran ser re-diseñados 0 descartados. Algunos estándares (ANSI Z353.3, 2011) definen un mínimo de $85 \%$ para un desempeño aceptable.

- Adicionalmente en contextos de emergencia, más del $5 \%$ en significados opuestos determina que el icono debe ser descartado del estándar.

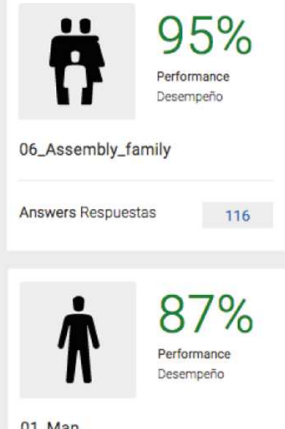

01_Man

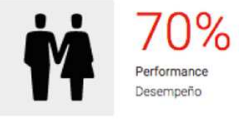

03_Assembly_couple

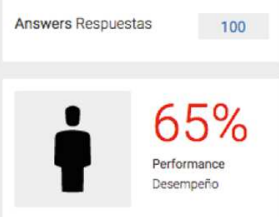

08_Boy

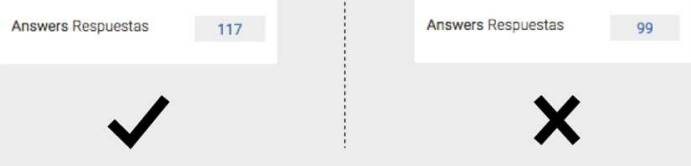

Figura 7: Ejemplos de íconos aceptados y no aceptados en los resultados en proceso del test de significados (mayo 2017)

\section{Proyecciones: Qué se espera lograr}

Hoy, la plataforma web asociada dispone un set de recursos accesibles para descargar, evaluar y eventualmente adoptar los íconos. A partir de la experiencia se puede analizar los significados asociados a la emergencia para definir qué se puede mejorar: pertinencia, lenguaje, contexto.

De esta forma, Guemil podría aplicarse en plataformas simples que permitan a las personas aprender o debatir sobre el riesgo y la emergencia antes, y no solo esperar a enfrentarse con la información en un momento crítico. Sin embargo, dado que es un proyecto en proceso de validación, se espera alcanzar resultados finales en la medida en que estos sean conocidos y evaluados por los mismos usuarios, lo que generará un índice de desempeño.

Como proyección general estos podrían validarse a partir de su adopción por organizaciones que lideran la gestión del riesgo y la emergencia (ej. gobiernos locales). Esto puede ocurrir en los ámbitos públicos o privados, y puede ser a escala local, regional o internacional. Para ello cada ícono aceptado como parte del estándar se validaría gracias a este índice de desempeño.

Otra proyección puntual apunta a que su adopción podría facilitar el desarrollo de nuevas soluciones de comunicación para la preparación, mitigación y recuperación de desastres, en distintos niveles de complejidad y alcance. Adicionalmente, en el espacio académico se proyecta divulgar: generar y enriquecer la colaboración internacional e interdisciplinar, parte de la misión original del proyecto.

Por cómo se ha llevado a cabo hasta ahora el diseño basado en testeo con usuarios, desde los resultados del proyecto se pueden también señalar algunas reflexiones que son expuestas como conclusión. Sin embargo, por sus características emocionales y la complejidad del contexto, un aspecto que es todavía muy difícil de probar o medir en su desempeño, es qué ocurre en el momento mismo de la emergencia (durante) y la 'tensión cognitiva' que se genera (Jaenichen, 2011). Eventualmente, tecnologías de simulación inmersivas como la realidad virtual o el diseño de experiencias basadas en gamificación son espacios abiertos de proyección para explorar nuevas aplicaciones.

\section{Reflexiones a modo de conclusión}

Como diseño de información, Guemil demuestra que es posible conducir iniciativas desde el ciclo del riesgo y la experiencia de usuario de forma diferente, aportando elementos visuales de apoyo y retroalimentación para contribuir al manejo del riesgo y emergencia. El proyecto permite debatir sobre cómo optimizar la comunicación desde la experiencia individual y aportar a la resiliencia de las comunidades desde el despliegue de información efectiva.

Es clave estandarizar contenidos y lenguaje formal, para que las herramientas visuales cumplan un rol efectivo en la gestión del riesgo de desastres. Aunque, también es necesario conocer qué necesitan las personas y qué interpretan en 
contextos disruptivos. Como se evidencia, los testeos desde la versión inicial de Guemil contribuyen a identificar percepciones sobre la comprensión del lenguaje visual en la emergencia, que se continuarán iterando según las respuestas obtenidas.

Considerando que los resultados del proyecto son parte de un proceso, finalmente se presentan algunas reflexiones y recomendaciones en construcción:

- Iniciativas abiertas permiten sumar en el campo de la investigación proyectual. Por lo tanto, difundir el proyecto permitiría incorporar su adopción por parte de organizaciones relacionadas y trabajar colaborativamente.

- Por las condiciones y desafíos que presentan escenarios futuros como el cambio climático, las migraciones o la educación adaptativa, entre otros, hay oportunidades de conducir proyectos centrados en preparación y respuesta a nivel local e internacional. Para ello las herramientas de comunicación multi-cultural son clave.

- En el futuro, diseñando plataformas gráficas estandarizadas, con objetivos y mediciones centradas en aprender y comprender, estas pueden contribuir a construir significados, identificar amenazas y asistir oportunamente en las decisiones.

- Guemil es un proyecto de I + D concreto basado en diseño, para un contexto donde existen evidentes demandas y espacios para su adopción. La validación del desempeño en los símbolos es una forma de entregar los indicadores que se requieren.

\section{Notas}

${ }^{1}$ Una definición literal de Guemil es 'greca'. Según Loncón (2011) el Ngümin simboliza el sistema de escritura, y representa el dominio de la ciencia, el conocimiento, la manufactura y la transformación.

${ }^{1}$ El documento completo con las Methodological Guidelines puede descargarse desde la plataforma GitHub:

https://github.com/Guemil/Guemil Project

\section{Referencias}

Brugger Ch. (1990), Advances in the international standardization of public information symbols, in Information Design Journal 6:1, Amsterdam: John Benjamins,Publishing

Ramírez R.; Ramírez S. (2012) Estudio y diseño sistema de pictogramas turísticos Sernatur, Revista Diseña, Escuela de Diseño UC, Santiago

Abdullah, R. \& Hübner, R. (2006) Pictograms, Icons, and Signs. London: Thames \& Hudson,
Allard, J.; Briones, M.; Gálvez, F.; Ramírez R.; Ramírez, S.; Zurob, C. (2014) El diseño que pasa inadvertido. Santiago: Pontificia Universidad Católica de Chile, Escuela de Diseño.

Black, A.; Luna, P.; Lund, O; Walker, S. Eds. (2017) Information Design Research and Practice, London: Routledge,

Frascara, J. (2011) Qué es el Diseño de Información. Buenos Aires: Ed. Infinito

Pettersson, R. (2002) Information design, an introduction; Amsterdam: John Benjamins Publishing

Capítulos de libro:

Brugger, Ch. (1999), Public Information symbols: a comparison of ISO testing procedures. Visual Information for Everyday Use. London: Taylor \& Francis,

Wogalter, M. (1999) Factors influencing the effectiveness of warnings. On Zwaga, H, Boersema, T; Hoonhut (eds), 'Visual Information for everyday use'. London: Taylor \& Francis

Wogalter, S. Wogalter, M. Kalsher, B. Glover, L. Magurno, A. (1999) Connecting jumper cables: the effectiveness of pictorial warnings; On Zwaga, H, Boersema, T; Hoonhut (eds), 'Visual Information for everyday use' London: Taylor \& Francis.

Online Journals, Magazines, Newspapers and Databases:

Jaenichen, C. (2011) Design for Evacuation: Improving City Evacuation Instructions. Parsons Journal for Information Mapping, NY. doi:N/A

Kuzano, K, Isumi, T. Nakatami, Y. (2014) Disaster Information Sharing System using Pictograms: Representation of Multidimensional Information. Proceedings of the World Congress on Engineering and Computer Science; San Francisco

Loncón, E. (2011) Campaña promueve uso de banderas de pueblos originarios. Retrieved from: https://goo.gl/sjLVKr

Olgyay, Nora (2001) Development \& testing of the IIID safety symbols system. doi: 10.1075/idj.10.2.05olg

Zender, M. \& Mejía, M. (2013) Improving Icon Design: Through Focus on the Role of Individual Symbols in the Construction of Meaning. Visible Language 47.1 doi:N/A

Zwaga, H; Mijksenaar, P. (2000) The development and standardization of Warning Symbols; The role of Design and Human Factors. Proceedings of the Human Factors and Ergonomics Society.

AIGA Symbols Standard (1979), retrieved from http://www.aiga.org/symbol-signs

ANSI Z353: American National Standard, Criteria for Safety Symbols (preview document), retrieved from: https://webstore.ansi.org/Previews/PREVIEW_ANSI+NEMA+Z53 5.3-2011.pdf

ISO 7010:2011, 3.7 (2011), Graphical symbols -- Safety colours and safety signs -- Registered safety signs, retrieved from http://www.iso.org/iso/home/store/catalogue_tc/catalogue_detail. htm?csnumber $=54432$

TorqAid DRMC (2016), retrieved from: http://www.torqaid.com/resources/

UNISDR Terminology (2015), retrieved from: https://www.unisdr.org/we/inform/terminology 